\title{
An Improved Method for Dipole Modeling in EEG-Based Source Localization
}

\author{
Fredrik Edelvik\#1, Björn Andersson\#2, Stefan Jakobsson ${ }^{\# 3}$, Stig Larsson ${ }^{\circ 4}$, \\ Mikael Persson*5, Yazdan Shirvany ${ }^{* 6}$ \\ \# Fraunhofer-Chalmers Research Centre, Chalmers Science Park \\ SE-41288 Göteborg, Sweden \\ $\left\{{ }^{1}\right.$ fredrik.edelvik, ${ }^{2}$ bjorn. andersson, ${ }^{3}$ stefan.jakobsson $\}$ @fcc. chalmers.se \\ ${ }^{\circ}$ Department of Mathematical Sciences, Chalmers University of Technology \\ SE-412 96 Göteborg, Sweden \\ ${ }^{*}$ Department of Signals and Systems, Chalmers University of Technology \\ SE-412 96 Göteborg, Sweden \\ $\left\{{ }^{4}\right.$ stig, ${ }^{5}$ mikael.persson, ${ }^{6}$ yazdan. shirvany\}@chalmers.se
}

\begin{abstract}
The inverse problem in EEG-based source localization is to determine the location of the brain sources that are responsible for the measured potentials at the scalp electrodes. The brain sources are usually modeled as current dipoles which lead to a singularity in the right-hand side of the governing Poisson's equation. Subtraction methods have been proposed as a remedy and in this paper an improved subtraction method for modeling the dipoles is presented. The accuracy is demonstrated for radial and tangential sources in layered sphere models and is to the best of the authors' knowledge superior to previous methods for superficial sources. An additional advantage is that it produces a right hand side with few non-zeros which is beneficial for efficient solution of the inverse problem.
\end{abstract}

Keywords - epilepsy; EEG; finite element method; source reconstruction

\section{Introduction}

Epilepsy is one of the most common neurological diseases and about 0.5 to $1 \%$ of the population suffers from it. Surgical therapy has become an important therapeutic alternative for patients with medically intractable epilepsy. Correct and anatomically precise localization of the epileptic focus is mandatory to decide if resection of brain tissue is possible.

The most important non-invasive diagnosis tool used at epilepsy surgery centers is electroencephalography (EEG). To find the brain sources, which are usually modeled as current dipoles, that are responsible for the measured potentials at the EEG electrodes on the scalp is an inverse problem. Inverse problems are in general more difficult to solve than direct problems mainly due to ill-posedness and non-linearity. In this case the data is perturbed by noise and the potentials measured on the scalp surface do not uniquely determine the location of the dipoles as many different dipole configurations can generate the same distribution of potentials on the scalp.

Methods for solving the inverse problem in EEG-based source localization are based on solutions of the corresponding forward problem, i.e. simulation of the potentials on the scalp for a given source. The associated differential equations are the quasi-static Maxwell equations, which reduce to a Poisson equation. The sources are electrolytic currents, usually modeled as point dipoles, which are activated during epilepsy. The dipoles introduce singularities in the right-hand side of the Poisson equation that need to be treated.

A major limitation in EEG-based source reconstruction has been the poor spatial accuracy, which is attributed to low resolution of previous EEG systems and to the use of simplified spherical head models for solving the inverse problem. EEG-based source localization is an active field of research [1, 2], but partly due to the mentioned shortcomings the computational techniques are not yet part of the standard pre-surgical diagnostic workup.

Realistic models of the human head are geometrically complex and the tissue conductivity is highly inhomogeneous and even anisotropic, which makes finite element methods (FEM) well suited. The critical issue is how to handle the computational complexity of FEM with regard to the inverse problem. As has been shown in recent publications by Wolters et al.[3, 4] this can be accomplished through the use of algebraic multigrid preconditioners, parallel computing, and not the least the concept of reciprocity which makes it possible to solve the forward problem for each electrode position rather than for each possible dipole position. A so-called lead field matrix can be computed during the preprocessing. The repeated solution of the forward problem in the iterative process is then efficiently accomplished by multiplication of the lead field matrix by the estimated source to produce the electrode potentials.

Source localization is heavily dependent on the choice of dipole model and several different alternatives have been suggested in the literature. According to the survey article[5], the subtraction method[6] is the most accurate. In this paper we present an improved subtraction method that reduces the non-zeros in the right-hand side (RHS) vector and in addition 
produces the same non-zero structure of the vector regardless of dipole position. This implies that only part of the lead field matrix needs to be stored and the solution of the inverse problem will therefore be much faster.

\section{Methods}

The characteristic frequencies of the signals in the $\mathrm{kHz}$ range and below make the capacitive and inductive effects of the tissue negligible. Therefore, the quasi-static approximation of Maxwell's equations for the potential $\Phi$ can be used.

If we denote the domain of interest as $\Omega$ (with boundary $\partial \Omega$ ) and let the tissue conductivity be $\sigma$, we have Poisson's equation

$$
\nabla \cdot(\sigma \nabla \Phi)=\nabla \cdot \mathbf{j}^{s} \text { in } \Omega
$$

subject to the conditions

$$
\begin{aligned}
\hat{\mathbf{n}} \cdot(\sigma \nabla \Phi) & =0 \text { on } \partial \Omega \\
\Phi\left(\mathbf{x}_{\text {ref }}\right) & =0 .
\end{aligned}
$$

The source current $\mathbf{j}^{s}$ is modeled by a mathematical dipole at position $\mathbf{x}_{0} \in \Omega$ with the moment $\mathbf{M} \in \mathbf{R}^{3}$,

$$
\mathbf{j}^{s}(\mathbf{x})=\mathbf{M} \delta\left(\mathbf{x}-\mathbf{x}_{0}\right) .
$$

The source has a singularity at $\mathbf{x}_{0}$ and is therefore difficult to model with standard finite elements. A subtraction method[6] is used to circumvent this problem, where the total potential is split into two parts,

$$
\Phi=\Phi^{\infty}+\Phi^{\mathrm{corr}} .
$$

The first part, $\Phi^{\infty}$, is the solution to Eq. (1) in an unbounded domain with constant conductivity $\sigma^{\infty}$,

$$
\Delta \Phi^{\infty}=\frac{\nabla \cdot \mathbf{j}^{s}}{\sigma^{\infty}} .
$$

The solution can in this case be formed analytically as

$$
\Phi^{\infty}(\mathbf{x})=\frac{1}{4 \pi \sigma^{\infty}} \frac{\mathbf{M} \cdot\left(\mathbf{x}-\mathbf{x}_{0}\right)}{\left|\mathbf{x}-\mathbf{x}_{0}\right|^{3}}
$$

An equation for $\Phi^{\text {corr }}$ can now be formed by subtracting Eq. (5) from (1),

$$
-\nabla \cdot\left(\sigma \nabla \Phi^{\text {corr }}\right)=\nabla \cdot\left(\left(\sigma-\sigma^{\infty}\right) \nabla \Phi^{\infty}\right) \text { in } \Omega,
$$

subject to the conditions

$$
\begin{aligned}
\hat{\mathbf{n}} \cdot\left(\sigma \nabla \Phi^{\text {corr }}\right) & =-\hat{\mathbf{n}} \cdot\left(\sigma \nabla \Phi^{\infty}\right) \text { on } \partial \Omega \\
\Phi\left(\mathbf{x}_{\text {ref }}\right) & =0
\end{aligned}
$$

This approach to the problem ensures that the RHS of Eq. (7) is non-singular in the case where $\sigma$ is constant in a small ball around $\mathbf{x}_{0}$ [4]. The original RHS of Eq. (1) has support only at $\mathbf{x}=\mathbf{x}_{0}$, whereas the RHS of Eq. (7) has support where $\nabla \sigma \neq \mathbf{0}$. To obtain a more compact support $\Omega$ is divided into two subdomains

$$
\Omega=\Omega^{\mathrm{c}} \cup \Omega^{\mathrm{t}}
$$

where $\Omega^{c}$ is the region with constant $\sigma^{\infty}$ containing the source. See Fig. 1 for a schematic view in two dimensions. In this region the correction potential is solved for as described above, and in $\Omega^{\mathrm{t}}$ the whole solution is sought at once. Using Eqs. (7) and (1) the new formulation reads,

$$
\begin{aligned}
-\nabla \cdot\left(\sigma^{\infty} \nabla \Phi^{\mathrm{c}}\right) & =0 \text { in } \Omega^{\mathrm{c}}, \\
-\nabla \cdot\left(\sigma \nabla \Phi^{\mathrm{t}}\right) & =0 \text { in } \Omega^{\mathrm{t}},
\end{aligned}
$$

subject to the conditions

$$
\begin{aligned}
\Phi^{\mathrm{c}}+\Phi^{\infty} & =\Phi^{\mathrm{t}} \text { on } \partial \Omega^{\mathrm{c}} \\
\hat{\mathbf{n}}^{\mathrm{c}} \cdot\left(\sigma^{\infty} \nabla\left(\Phi^{\mathrm{c}}+\Phi^{\infty}\right)\right) & =\hat{\mathbf{n}}^{\mathrm{c}} \cdot\left(\sigma \nabla \Phi^{\mathrm{t}}\right) \text { on } \partial \Omega^{\mathrm{c}}, \\
\hat{\mathbf{n}}^{\mathrm{t}} \cdot\left(\sigma \nabla \Phi^{\mathrm{t}}\right) & =0 \text { on } \partial \Omega^{\mathrm{t}} \\
\Phi\left(\mathbf{x}_{\mathrm{ref}}\right) & =0 .
\end{aligned}
$$

In this formulation the support is only in $\partial \Omega^{\mathrm{c}}$, which can greatly improve efficiency when solving the forward problem multiple times.

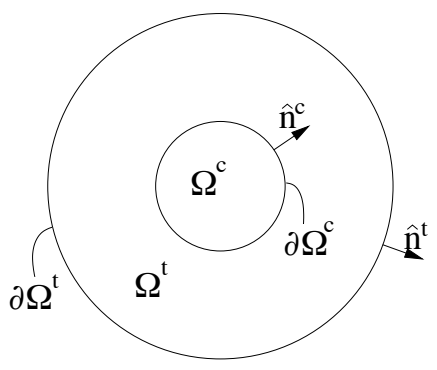

Figure 1: Schematic view of a two layer model.

The discretization of Eq. (10) performed by the piecewise linear FEM with basis functions $\varphi_{i}$ centered at the mesh points $\xi_{i}$. The solution to this equation, as well as $\Phi^{\infty}$, is represented in the FE space as

$$
\begin{aligned}
\Phi^{\infty} & \approx \sum_{\xi_{i} \in \Omega^{\mathrm{c}}} u_{i}^{\infty} \varphi_{i}(\mathbf{x}) \\
\Phi^{\mathrm{t}} & \approx \sum_{\xi_{i} \in \Omega^{\mathrm{t}}} u_{i}^{\mathrm{t}} \varphi_{i}(\mathbf{x}) \\
\Phi^{\mathrm{c}} & \approx \sum_{\xi_{i} \in \Omega^{\mathrm{c}} \wedge \xi_{i} \notin \partial \Omega^{\mathrm{c}}} u_{i}^{\mathrm{c}} \varphi_{i}(\mathbf{x})+\sum_{\xi_{i} \in \partial \Omega^{\mathrm{c}}}\left(u_{i}^{\mathrm{t}}-u_{i}^{\infty}\right) \varphi_{i}(\mathbf{x}) .(12 \mathrm{c})
\end{aligned}
$$


After applying variational and FEM techniques to Eqs. (10) and (11) we arrive at the system of linear equations

$$
\mathbf{K u}=\mathbf{j},
$$

where $\mathbf{u}$ contains the nodal degrees of freedom in both domains, $\Omega^{\mathrm{c}}$ and $\Omega^{\mathrm{t}}$. The stiffness matrix has the entries

$$
K_{i, j}=\iiint_{\Omega}\left(\sigma \nabla \varphi_{i}\right) \cdot \nabla \varphi_{j} \mathrm{dV} .
$$

The vector $\mathbf{j}$ has contributions from Eqs. (11a) and (11b)

$$
\mathbf{j}=\mathbf{j}_{D}+\mathbf{j}_{N}
$$

The first part can be written as

$$
\mathbf{j}_{D}=\mathbf{K}_{D} \mathbf{u}^{\infty},
$$

where the entries in $\mathbf{K}_{D}$ are given by

$$
\begin{aligned}
K_{D_{i, j}}=\iiint_{\Omega}\left(\sigma^{\infty} \nabla \varphi_{i}\right) \cdot \nabla \varphi_{j} \mathrm{dV}, \\
\xi_{i} \in \Omega^{\mathrm{c}} \wedge \xi_{i} \notin \partial \Omega^{\mathrm{c}}, \xi_{j} \in \partial \Omega^{\mathrm{c}} .
\end{aligned}
$$

The entries in the vector $\mathbf{j}_{N}$ are given by

$$
j_{N_{i}}=\iint_{\partial \Omega^{\mathrm{c}}} \varphi_{i} \hat{\mathbf{n}}^{\mathrm{c}} \cdot\left(\sigma^{\infty} \nabla \Phi^{\infty}\right) \mathrm{dS} .
$$

The total potential in $\Omega$ can then be computed by means of Eq. (4).

As a comparison, if the standard subtraction method[6] is used to discretize Eqs. (7) and (8) on a multilayer isotropic sphere with different conductivity in each layer, the RHS vector would consist of contributions of the type

$$
j_{i}=\iint_{S_{k}} \varphi_{i} \hat{\mathbf{n}} \cdot\left(\left(\sigma^{k+1}-\sigma^{k}\right) \nabla \Phi^{\infty}\right) \mathrm{dS},
$$

for each interface $k$.

The system of linear equations, Eq. (13), can be efficiently solved iteratively by a conjugate gradient (CG) solver preconditioned by an algebraic multigrid (AMG) method.

\section{Results}

The model used for the head is a four layer sphere which has been used in several studies [5]. The parameters are defined in Table 1. The validation and comparison of the method was done by calculating the potential for dipoles with eccentricity from 0 to $97.4 \%$, where the eccentricity of the dipole is defined as the ratio between the distance from the center of the sphere to the source divided by the radius of the brain compartment, $78 \mathrm{~mm}$. Comparisons are made for dipoles directed in the two principal directions, normal and tangential to the surface. Finally, calculations were performed on two tetrahedral meshes of different resolution.

Table 1: Parameterization of the four layer sphere model

\begin{tabular}{|c|c|c|c|c|}
\hline & Brain & CSF & Skull & Scalp \\
\hline Outer shell radius [mm] & 78 & 80 & 86 & 92 \\
\hline Conductivity [S/m] & 0.33 & 1.0 & 0.0042 & 0.33 \\
\hline
\end{tabular}

The errors are calculated by comparing the solutions to the potential given by an analytic solution[7] at each surface node. The maximum relative error, the relative error, the relative difference measure, and the magnification factor, are defined as

$$
\begin{aligned}
e_{\max } & =\max _{i}\left|u_{i}^{a}-u_{i}^{n}\right| / \max _{i}\left|u_{i}^{a}\right|, \\
e_{\mathrm{RE}} & =\frac{\left\|\mathbf{u}^{a}-\mathbf{u}^{n}\right\|_{2}}{\left\|\mathbf{u}^{a}\right\|_{2}}, \\
e_{\mathrm{RDM}} & =\sqrt{\sum_{i}\left(\frac{u_{i}^{a}}{\left\|\mathbf{u}^{a}\right\|_{2}}-\frac{u_{i}^{n}}{\left\|\mathbf{u}^{n}\right\|_{2}}\right)^{2}} \\
e_{\mathrm{MAG}} & =\frac{\left\|\mathbf{u}^{n}\right\|_{2}}{\left\|\mathbf{u}^{a}\right\|_{2}}
\end{aligned}
$$

where $u_{i}^{a}$ is the value of the analytical solution at surface node $i, u_{i}^{n}$ is the corresponding value of the numerical solution. $\|\cdot\|_{2}$ denotes the Euclidean norm.

To benchmark the method proposed is this paper we compare with the state-of-the-art method in the literature[6] according to the survey article[5]. The errors are shown in Fig. 2 where the two methods are compared on two meshes of different resolution. The upper subfigure shows the result for radial dipoles and the lower the result for tangential dipoles. The newly developed method shows significantly better results for dipoles placed close to the surface of the inner layer. For the coarser mesh the ratio of the RDM error between the two solution methods at eccentricity $97.4 \%$ is 5.3 for both radial and tangential dipoles. The corresponding ratios for the finer mesh are 3.3 , and 2.6, respectively. The largest improvement is indicated in the relative maximum error. On the coarser mesh the ratio of the errors are 22 and 25 for radial and tangential dipoles, respectively. The corresponding values for the finer mesh are 14 and 13, respectively. 

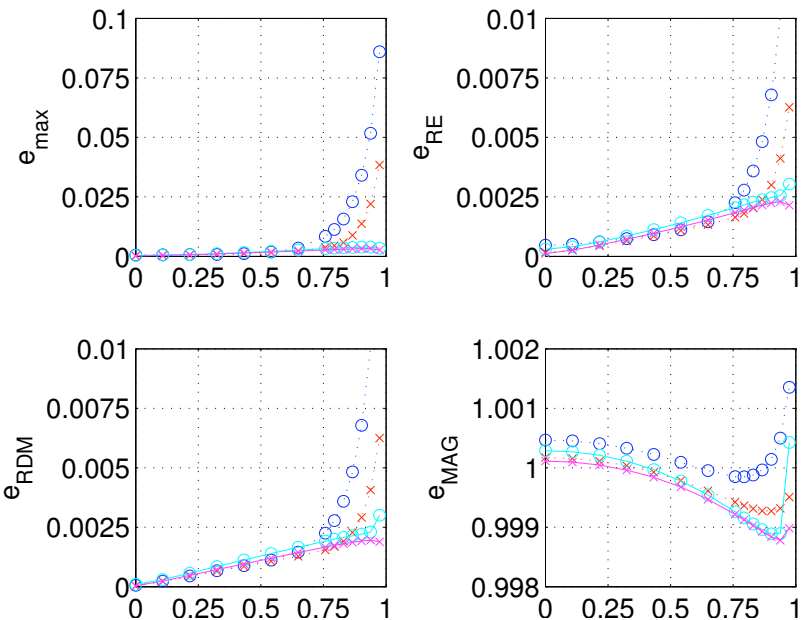

(a) Radial dipole
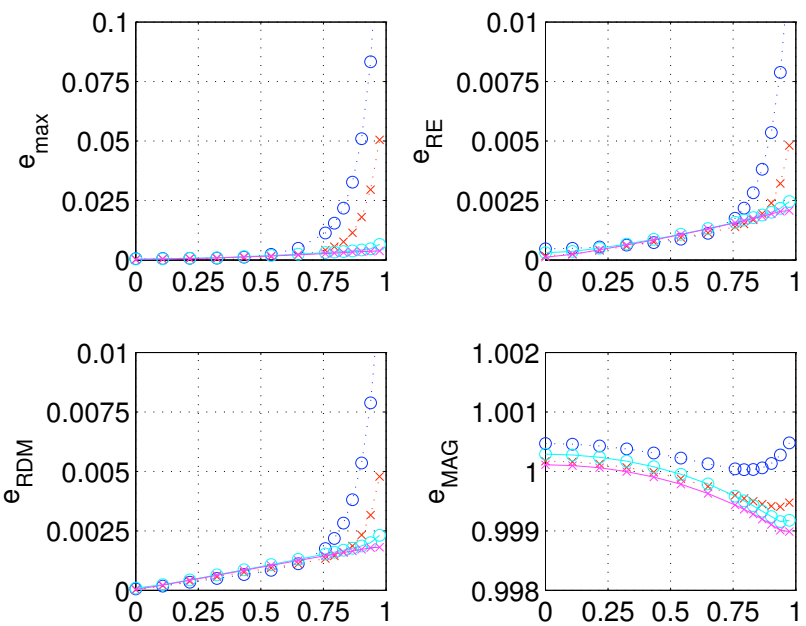

(b) Tangential dipole

Figure 2: Error as a function of eccentricity. Full lines: method presented here, dotted lines: subtraction method from [6].○ markers: mesh with $233 \mathrm{k}$ nodes, $\times$ marker: mesh with $991 \mathrm{k}$ nodes.

\section{Conclusions}

In this paper we have presented an improved method for modeling the dipole in source localization. The proposed method has been shown to be more accurate than the stateof-the-art method in the literature. Additional advantages are that the RHS has fewer non-zeros and the same non-zero structure regardless of dipole position something which in contrast to earlier approaches also holds for anisotropic tissue. This fact will be very beneficial for efficient solution of the inverse problem. Future work will include development of methods for solving the inverse problem and application to source localization with realistic head models generated from a segmentation of the patient's MRI.

\section{Acknowledgement}

This work was supported in part by the Swedish Foundation for Strategic Research (SSF) through the Gothenburg Mathematical Modeling Centre (GMMC).

\section{References}

[1] H. Hallez, B. Vanrumste, R. Grech, J. Muscat, W. De Clercq, A. Vergult, Y. D’Asseler, K. P. Camilleri, S. G. Fabri, S. Van Huffel, and I. Lemahieu. Review on solving the forward problem in EEG source analysis. Neuroengineering and Rehabilitation, 46(4), 2007.

[2] C. M. Michel, M. M. Murray, G. Lantz, S. Gonzalez, L. Spinelli, and R. Grave de Peralta. EEG source imaging. Clinical Neurophysiology, 115(10):2195-2222, 2004.

[3] C. H. Wolters, M. Kuhn, A. Anwander, and Reitzinger S. A parallel algebraic multigrid solver for finite element method based source localization in the human brain. Computing and Visualization in Science, 5(3), 2002.

[4] C. H. Wolters, H. Köstler, C. Härdtlein J. Möller, L. Grasedyck, and W. Hackbusch. Numerical mathematics of the subraction method for the construction of a current dipole in EEG source reconstruction using finite element head models. SIAM J. Scientific Computing, 30(1):24-45, 2007.

[5] P. H. Schimpf, C. Ramon, and J. Haueisen. Dipole models for the EEG and MEG. IEEE Trans. Biomedical Engineering, 49(5):409-418, May 2002.

[6] S. van den Broeh, H. Zhou, and M. Peters. Computation of neuromagnetic fields using finite-element method and Biot-Savart law. Medical and Biological Engineering and Computing, 34(1):21-26, 1996.

[7] M. Sun. An efficient algorithm for computing multishell spherical volume conductor models in EEG dipole source localization. IEEE Trans. Biomedical Engineering, 44(12):1243-1252, Dec. 1997. 